\title{
Statistics of firn closure: a simulation study
}

\author{
I. G. ENTING \\ CSIRO Division of Atmospheric Research, Mordialloc, Victoria 3195, Australia
}

\begin{abstract}
The trapping of bubbles of air in polar ice has provided a unique record of past atmospheric composition. However, the interpretation of measured concentrations depends on the statistics of the trapping process. Measurements of trace atmospheric constituents whose concentrations are changing steadily can be interpreted in terms of an "effective age" of the gas which differs from the age of the ice by a delay which corresponds to the mean trapping time. The statistics of bubble trapping can be modelled as a percolation model which is one of a class of models whose transitions are characterized by large critical fluctuations. These critical fluctuations cause an intrinsic sample-to-sample variability in the delay time and thus in the effective age. Monte Carlo simulations using a lattice model of the firn are presented, showing the effect of finite sample size on the age distribution of trapped gas. For samples containing more than about $10^{3}-10^{4}$ bubbles, the simulations indicate that the range of variability is small compared to the average duration of the trapping process.
\end{abstract}

\section{INTRODUCTION}

The present paper concerns the trapping of air bubbles as firn (compacted but permeable accumulated snow grains) becomes non-permeable ice as the density increases. The bubble trapping is analysed using the percolation model from lattice statistics. The statistics of this air-bubble trapping are of some importance because the composition of air from bubbles in polar ice provides a record of atmospheric concentrations of climatically active atmospheric constituents such as $\mathrm{CO}_{2}$. These records span periods ranging from decades to millennia, depending on the accumulation rate and thickness of the ice cap from which the record is obtained (see, for example, Delmas and others, 1980; Neftel and others, 1985; Pearman and others, 1986; Barnola and others, 1987). As well as being required for the direct interpretation of concentrations of trapped gases, the statistics of the firn-closure process are also important in the interpretation of those measurements of firn closure that are to be used in the interpretation of gas data.

The percolation model is a statistical model describing random networks. It has been used to model a wide range of physical processes (Zallen, 1983). The relevance of the percolation model to bubble trapping in firn was recognized by Enting (1985b) and Stauffer and others (1985). When modelling firn closure in terms of percolation, we can draw on a very large body of existing work on this and related models. Section 2 below reviews some key results from this field of "lattice statistics" and introduces some of the technical terms involved (see also the review by Enting (1987)). The present study is motivated by the fact that many of these lattice-statistics models, including the percolation model, exhibit transitions characterized by fluctuations on all length scales. Enting (1985b) identified the fluctuations in the trapped bubble volume, as measured by Schwander and Stauffer (1984), with the statistical fluctuations of the percolation transition. In order to investigate the significance of these fluctuations for gas dating, we performed Monte Carlo simulations using the ultramarine lattice model introduced by Stauffer and others (1985).

The percolation model, as used here, is a descriptive statistical model of firn closure, rather than being fully mechanistic. In particular, the model is expressed in terms of the probabilities of channel closure and does not attempt to predict these from the physical properties of the firn. This approach is motivated by the recognition that the statistical aspects are the most important characteristics of the firn-closure process. Any more comprehensive model that seeks to predict properties such as the close-off density must take the percolation statistics into account as a prerequisite for defining the close-off point. Some of the issues involved in modelling firn closure have been discussed by Stauffer (1982). The universality theory property of lattice model transitions (see section 2 below) predicts that, suitably scaled, a simple percolation model will correctly represent the characteristics of more complicated percolating systems.

One important qualitative result from this identification was the recognition that, because of the sharp cutoff in the trapping, the deconvolution of atmospheric composition from a record of mean bubble composition is a relatively well-conditioned inversion problem (Enting, 1985a). Enting and Mansbridge (1985) (henceforth referred to as EM) suggested that, because of this relatively well-conditioned nature of the process of 
deconvolving bubble compositions to obtain a record of atmospheric composition, the trapping could be parameterized by an effective age. This effective age differed from the age of the ice by a delay time which was expressed as an integral over the trapping distribution (see Equation (6) below). From the simulations described below, we can estimate the sample-to-sample variation in this delay time.

The lay-out of the remainder of the paper is as follows. Section 2 reviews some key concepts from lattice statistics. Section 3 reviews the concepts of the bubble-trapping distribution and the effective age of the gas. A number of relevant measurements of ice-core properties are noted. Section 4 describes the lattice model used in the Monte Carlo studies. Section 5 presents the results of the simulations. Section 6 gives a brief discussion of the effect of pore closure on the restriction of diffusive mixing through the firn, and the role of percolation theory in understanding this effect. Section 7 gives some concluding remarks while additional details of the simulation algorithm are given in the Appendix.

\section{CRITICAL PHENOMENA AND PERCOLATION}

In this section, we review some of the relevant results from lattice-statistics models of critical phenomena, with particular reference to the percolation model. Further details may be found in Enting (1987) and references therein. Some technical terms of particular relevance to the firn-closure problem are noted below in italics.

The term lattice statistics is used to cover a range of mathematical or statistical models defined at discrete points in space, usually on a regular lattice. The properties of the models are derived from locally defined statistical characteristics. Many of the applications have been in solid-state physics where the lattice corresponds to an actual crystal lattice and the statistical variations arise from the effects of temperature. These models have been the subject of very extensive study. The references in the review by Enting (1987) can be used as an initial point of contact with the lattice-statistics literature. In addition, Efros (1986) and Stauffer (1985) have published introductory books specifically on the percolation model. The first of these covers a greater range of topics but suffers from the lack of any references.

The percolation model has a very simple statistical characterization. In the form used to model bubble trapping, the bubbles are associated with points on the lattice, the sites. Neighbouring sites are regarded as being linked by bonds on the lattice. These bonds are regarded as being either "connected" or "broken". The connectivity is assigned randomly, independently for each bond. The model deals with the statistics of clusters of sites joined by chains of "connected" bonds. When modelling firn closure, the "connected" bonds represent open pores or channels between the snow grains.

There is a wide class of lattice models (including percolation models) which exhibit what are known as critical phenomena. The characteristics of these critical phenomena are:

i. There is a critical probability at which the macro- scopic properties of the system change abruptly. In the percolation model, there is a particular (latticedependent) probability, $p_{\mathrm{c}}$, above which arbitrarily large connected clusters will occur, but below which the mean cluster size remains finite. In other lattice models, the abrupt change is used to model a change in thermodynamic phase as a function of temperature. The general term critical point is used to define the point at which the change occurs.

ii. At this critical point, there is no characteristic length scale; the spatial characteristics show statistical self-similarity (i.e. various statistical properties remain invariant under a change of length scale). In the terminology of Mandelbrot (1977), clustering properties are described by fractals. Correlations between sites show power-law dependence on distance at the critical point.

iii. Various other statistical properties exhibit singularities at the critical point. The behaviour in approaching the critical point can often be represented by power laws. The powers involved are called the critical exponents with standard symbols (usually Greek letters) denoting many of these exponents. For example, as a critical point is approached, characteristic length scales (denoted $\xi$ ) diverge with a powerlaw dependence on the distance from the critical point: $\xi \propto\left|p-p_{\mathrm{c}}\right|^{-\nu}$. Such power-law scaling relations apply within a critical region surrounding the critical point.

iv. The values of the critical exponents are independent of many local details of the statistical model. In particular, for percolation models, the critical exponents are the same, whether one considers clusters of randomly occupied sites, clusters of randomly occupied bonds or, as in the model described below, clusters of sites connected by randomly occupied bonds. The critical exponents are also independent of the particular lattice, apart from depending on the dimensionality. These independence properties are referred to as universality. An extreme example is the use of a lattice model to represent the liquid-gas coexistence line and the liquid-gas critical point that terminates this co-existence line. This model can represent many features of such critical points even though neither phase occurs on a lattice.

v. As the critical point is approached, various statistical quantities become extremely sensitive to small perturbations. In the liquid-gas model, the compressibility becomes arbitrarily large. In magnetic systems, the susceptibility (the rate of change in magnetization as the external field is increased) diverges. In the percolation model, there is an analogous divergence in the mean number of sites per connected cluster.

vi. As well as the large responses to external forcing, the statistical properties near the critical point exhibit large intrinsic fluctuations. In the liquid-gas system, these correspond to density fluctuations that are 
observed through anomalously high light scattering, known as critical opalescence. Similar statistical fluctuations occur in the percolation model. Enting (1985b) interpreted the highly variable results for the amount of trapped gas near the firn close-off (as measured by Schwander and Stauffer (1984)) as an example of such critical fluctuations. These observations were a key piece of evidence in arguing for the applicability of the percolation model in representing bubble trapping in firn. These critical fluctuations are an intrinsic part of the transition. Indeed, critical phenomena are sometimes referred to as co-operative phenomena because the transitions occur due to the cooperative effects of such fluctuations.

The most common forms of percolation model are site percolation (clusters of randomly occupied sites where pairs of occupied neighbours are regarded as connected) and bond percolation (clusters of randomly occupied bonds). Stauffer and others (1985) modelled firn closure using a conventional bond percolation model. Enting (1986, 1987) proposed that, to represent bubble trapping, a more appropriate form of the percolation model would consider clusters of sites connected by randomly occupied bonds. In this "bubble-trapping" percolation model, the sites represent space between ice grains and the bonds represent connecting channels that are gradually closed off. A key statistic in percolation modelling is the percolation probability, $P$, which is the probability of a site or bond being part of an infinite connected cluster. At the critical point, $P$ goes to zero as $P \propto\left|p-p_{\mathrm{c}}\right|^{\beta}$ with $\beta \approx 0.454$ in three dimensions.

Enting (1986, 1987) noted that the bubble-trapping model (clusters of sites connected by randomly occupied bonds) was the special form of a percolation model obtained by taking the $q \rightarrow 1$ limit of the $q$-state Potts model (Fortuin and Kastelyn, 1972; Wu, 1978). This connection showed the relation between the percolation model and more general statistical mechanics models of phase transitions. It was also useful in the derivation of series expansions for the trapping distribution (Enting, 1986), but otherwise gives little insight into the percolation model and so will not be considered further in this paper.

Enting (1987) listed five main classes of mathematical technique that have been used to investigate critical phenomena in lattice models. These are (a) exact solutions (generally not available), (b) statistical closures (usually inaccurate in the critical region), (c) power-series expansions of statistical moments (as applied to firn closure by Enting (1986)), (d) renormalization group techniques and (e) Monte Carlo simulations.

Monte Carlo simulations are used in the present study because the statistical fluctuations are simulated directly. However, they are restricted by the fact that the simulation can only be for a finite system. The other computational techniques above allow a formal limit of arbitrarily large systems to be considered. Since the range of spatial correlation diverges near the critical point, finite-size effects become important and must be corrected for.

An example of the importance of correcting for finitesize effects is that Enting (1986) estimated the $p_{\mathrm{c}}=$
$0.39 \pm 0.01$ using series expansions based on combinatorial factors that involved less than 20 sites, but which were not subject to edge effects. In contrast, the Monte Carlo calculation by Stauffer and others (1985) estimated $p_{\mathrm{c}}=0.42$ on the basis of simulations involving over 4000 sites. The results are presented below, involving up to 165888 sites, show that the apparent $p_{\mathrm{c}}$ decreases with sample size and appears to be converging towards a value in the range estimated from the series calculations.

Since the main object of the present paper is an analysis of the size-dependence of the sampling statistics of bubble trapping, it is necessary to distinguish this from the finite-size effects noted above. Finite-size effects inevitably occur in Monte Carlo simulations because only a finite number of sites can be simulated. Within such finite simulations, we consider the question of how the bubble-trapping statistics are affected by taking specific sized samples for gas analysis. In order to study this second type of finite-size effect, the statistics are taken from a sub-region of the sites simulated.

\section{THE BUBBLE-TRAPPING DISTRIBUTION}

In the analysis of bubble trapping, there are a number of alternatives for the independent variable. The percolation models work in terms of the probability of bond closure $(u)$ while levels within an ice core can be described in terms of density $(\rho)$, depth $(d)$ or age $(z)$. In ideal situations, the four variables $u, \rho, d$ and $z$ are all monotone increasing functions of each other. Results reviewed by Schwander and Stauffer (1984) suggest that the critical probability $u_{c}$, beyond which trapping is complete, corresponds to $\rho_{\mathrm{c}}=0.82 \mathrm{Mg} \mathrm{m}^{-3}$ under a range of different deposition rates. There is, however, a dependence of closure density on the temperature of deposition (personal communication from D. Raynaud). Further studies of aspects of firn closure have been given by Stauffer (1982), Loosli (1983), Stauffer and others (1985), Schwander and others (1988) and Schwander (1988).

The relation between depth density and the age of the ice depends on the local snow-accumulation rate which varies by several orders of magnitude between different sites. The relationship between depth and age can be established in a variety of ways. In regions of high accumulation, seasonal variations in quantities such as electrical conductivity or oxygen-isotope ratios can be used to count the number of years since deposition. In regions of slower accumulation, ages must be inferred indirectly by using glaciological models.

EM (i.e. Enting and Mansbridge, 1985) described the basic concepts involved in interpreting the mean concentrations of gases recovered from ice-core samples. They defined $\bar{R}\left(z, z_{\mathrm{t}}\right)$ as the amount of gas that was trapped $z_{\mathrm{t}}$ years ago in ice that was deposited $z$ years ago. Clearly, this is zero for $z_{\mathrm{t}}>z$, i.e. the gas cannot be trapped until the ice is present. The existence of a sharp transition between firn and ice implies that there is also a lower bound on $z_{\mathrm{t}}$. The gas must be trapped within a time of $z_{\mathrm{c}}$ of the snowfall. Thus, for non-zero $R$, we require

$$
z-z_{\mathrm{c}}<z_{\mathrm{t}}<z .
$$


If we assume a uniform deposition process, then we set

$$
\bar{R}\left(z, z_{\mathrm{t}}\right)=R\left(z-z_{\mathrm{t}}\right) .
$$

The sharpness of this trapping distribution determines the extent to which gas bubbles from different times are trapped in a single layer. The cumulative trapping distribution was defined by EM as

$$
S(z)=\int_{0}^{z} R\left(z^{\prime}\right) \mathrm{d} z^{\prime}
$$

They used this to define the normalization by requiring

$$
S\left(z_{\mathrm{c}}\right)=1 \text {. }
$$

EM showed that, if ambient gas concentrations $c(z)$ were changing linearly, then the average concentration, $q(z)$, occurring in bubbles in an ice layer of age $z$ corresponded to ambient concentrations at a younger age, $z_{\text {eff }}$, so that

$$
q(z)=c\left(z_{\text {eff }}\right), \quad \text { so long as } z>z_{\mathrm{c}}
$$

with $z_{\text {eff }}$ defined in terms of the mean delay or mean trapping time $\bar{z}$ by:

$$
\bar{z}=z-z_{\text {eff }}=\int_{0}^{z_{\mathrm{c}}} z^{\prime} R\left(z^{\prime}\right) \mathrm{d} z^{\prime}=z_{\mathrm{c}}-\int_{0}^{z_{\mathrm{c}}} S\left(z^{\prime}\right) \mathrm{d} z^{\prime} .
$$

In its simplest form, neglecting finite-size effects, the percolation model of bubble trapping equates $1-S(z)$ with the percolation probability, $P$. For an infinite lattice, percolation theory defines $P$ as the probability that a given site is part of an infinite cluster of sites connected by open channels. For finite samples, the corresponding quantity will exhibit statistical fluctuations as shown in the simulations presented below. In view of the analysis of gas ages summarized above, the most important question concerning critical fluctuations is the size of the critical fluctuations in the mean trapping time $\bar{z}$. It should be noted that since, by definition,

$$
S(z)=1 \quad \text { for } z \geq z_{\mathrm{c}}
$$

we have

$$
\bar{z}=z_{x}-\int_{0}^{z_{x}} S\left(z^{\prime}\right) \mathrm{d} z^{\prime} \quad \text { for } z_{x}>z_{\mathrm{c}}
$$

independently of $z_{x}$.

When dealing with finite samples, the point of complete trapping will show fluctuations about the infinite size limit $z_{\mathrm{c}}$. For this reason, $z_{x}$ must be chosen to be greater than the actual point of complete trapping for any sample under consideration.

The analysis summarized above describes concentrations, $q(z)$, for layers in which the air is completely trapped. For concentrations, $q(z)$, obtained in ice layers that are not completely closed off from the atmosphere, the expressions above must be modified. What is required (see EM) is to rescale $S(z)$ so that it describes incomplete trapping. The age, $z$, of the ice layer replaces $z_{\mathrm{c}}$ in Equation (6) to give

$$
\bar{z}=z-S(z)^{-1} \int_{0}^{z} S\left(z^{\prime}\right) \mathrm{d} z \quad \text { for } z<z_{\mathrm{c}} .
$$

This aspect of the model will not be considered in the present study.

\section{THE LATTICE MODEL}

The application of the percolation model to firn closure is based on the following propositions:

i. The snow grains of the firn form a random network of spaces and channels containing air.

ii. As the firn is compressed, the narrowest gaps are closed, leaving bubbles of air in the larger spaces. A more detailed analysis of the changes occurring during firnification has been given by Stauffer (1982).

iii. The closure of channels will be effectively a random process reflecting the random structure of the firn.

iv. The percolation model is the appropriate tool for analysing the statistics of random closures. The various properties described in section 2 above will apply.

v. Percolation modelling predicts a sharp transition between the state where the firn is permeable and the impermeable ice. This transition reflects the longrange statistics of connectivity and will occur before all channels are closed.

vi. The universality property of percolation models predicts that the main features of the bubble trapping at the firn-to-ice transition will not depend on the details of the model. The only effect of changes in such details will be a smooth rescaling of model parameters. Among such "irrelevant" details are: (a) the use of a regular lattice rather than a random network, (b) local correlations in channel closure, (c) assigning all of the trapped volume to the larger spaces that become bubbles and ignoring the air in the channels.

vii. While universality enables us to relate critical behaviour on different lattices, the comparisons will be most direct if the choice of lattice reflects the statistics of connectivity in the firn. On the basis of measurements of firn structure (see Stauffer, 1982), Stauffer and others (1985) suggested that an appropriate lattice model for bubble trapping could be obtained by taking the ice grains as being on a body-centredcubic (b.c.c.) lattice.

viii. The statistics will exhibit large fluctuations near the firn-to-ice transition.

ix. If the trapping times are sufficiently long compared to the times of mixing through the firn, then the age distribution of the trapped gas will correspond to the age distribution of the time of trapping.

x. The percolation model considers the percolation probability, $P$. This is the probability that a given site will be part of an infinite cluster of connected sites rather than part of a finite cluster. The bubbletrapping model regards sites in finite clusters as being cut off from the surface and so the proportion of trapped bubbles is $1-P$. 
xi. The function $1-P(u)$ gives the mean proportion of trapped bubbles in an infinite system. Enting (1986) applied series-expansion techniques in estimating this function. The present paper considers the extent to which the proportion of trapped bubbles in finite samples will show a sample-to-sample variability about the mean.

xii. The most appropriate way to study the statistics of bubble trapping is through Monte Carlo simulation of a limited region of firn.

Following the earlier work by Stauffer and others (1985) and Enting (1986), we model the firn by locating snow grains on a b.c.c. lattice with the interstitial sites representing the locations of air bubbles. The sites each are joined to their four nearest neighbours by bonds that are connected (with probability $p$ ) or broken (with probability $u=1-p$ ). We describe the lattice in terms of cubic cells containing 12 sites and 24 bonds as shown in Figure 1, and consider arrays of $m \times m \times n$ of these cubic cells. We consider the statistics of connection to the upper boundary (layer 1) as an approximate representation of long-range connection to the surface. We apply periodic boundary conditions in the horizontal $(m \times m)$ layers, i.e. each horizontal cross-section is taken as being surrounded by identical copies of itself. The simulation procedure assigned each bond (connecting sites $i$ and $j$ ) a randomly chosen "cut-off time", $u_{i j}$, which corresponded to the "time" at which bond $(i, j)$ closed. The $u_{i j}$ are described as "times" in order to give a more intuitive description but are more correctly regarded as the probabilities of site trapping and bond closure and

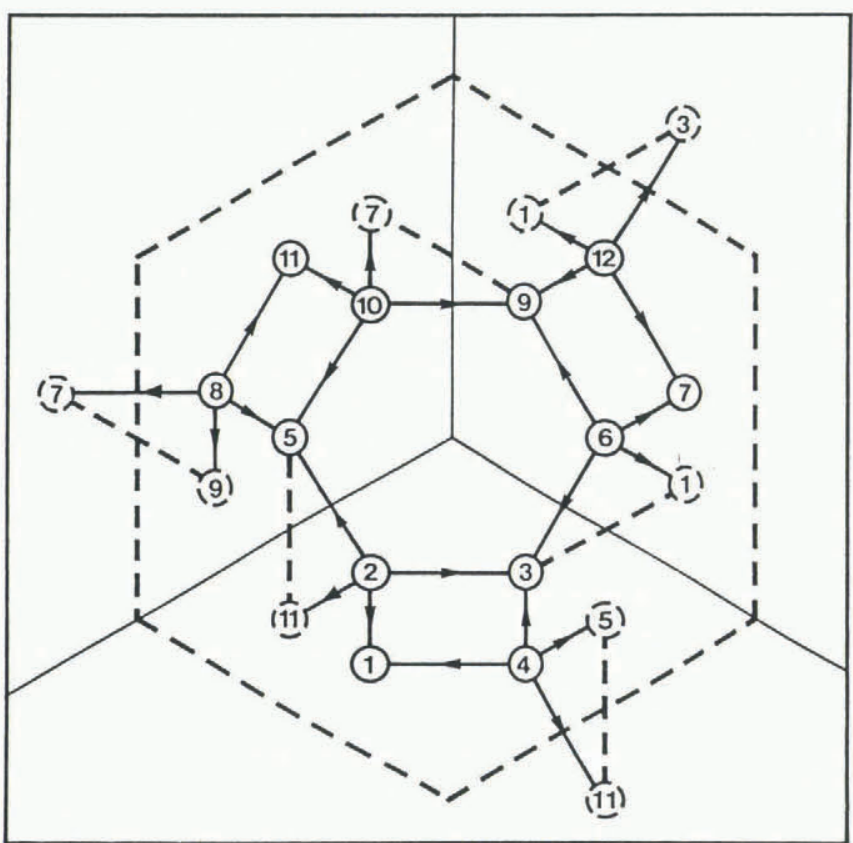

Fig. 1. The basic simple cubic cell and the 12 sites and 24 bonds defining the lattice of b.c.c. interstitial sites. Each site lies on one of the square faces defined by two of the axes and two of the dashed lines. Dashed sites are regarded as lying in cells neighbouring the one shown. therefore the $u_{i j}$ were selected uniformly from the range $[0,1]$.

The model statistics of bubble trapping were obtained from the site-closure values $u_{i}$, the "time" beyond which site $i$ was no longer connected to the surface, i.e. the "time" at which the last path of open bonds connecting site $i$ to the surface was closed at some point. These $u_{i}$ were calculated from the $u_{i j}$ using the algorithm described in the Appendix. The bubble-trapping statistics were computed using a sub-set, $A$, of sites so as to reduce the influence of the finite size of the simulation. Specifically, the bubble-trapping distribution was

$$
S(u)=|A|^{-1}|X(u)|
$$

where

$$
X(u)=\left\{u_{i}: i \in A \text { and } u_{i} \leq u\right\} .
$$

Consequently $X(u)$ is the number of sites cut off from the upper boundary (i.e. trapped) at "time" $u$ and $S(u)$ is the proportion of trapped sites at "time" $u$.

The lattice model involves several key approximations:

(i) It regards all the trapped gas as being in the sites rather than being partly in the bonds.

(ii) It assumes independent closure probabilities for each bond.

(iii) It assumes a uniform lattice rather than a random structure.

(iv) It applies only to uniform deposition conditions.

The most important of these approximations is the assumption of uniform snow-deposition conditions. This is because, as noted in section 2 (point v), co-operative phase transitions such as the percolation transition, are characterized by divergent susceptibilities to external perturbations as well as divergences in the intrinsic fluctuations. The analysis of externally forced variations is largely outside the scope of this paper, except to note that changing conditions will mainly act to change $z_{\mathrm{c}}$ by changing the relation between $z$ and $u$. Since the infinite size limit of $S(z)$ has an infinite gradient at $z_{\mathrm{c}}$, point-wise values of the trapping distribution near $z_{\mathrm{c}}$ can have finite responses to arbitrarily small perturbations. However, a change, $\Delta\left(z_{\mathrm{c}}\right)$, in the critical point will induce only a change of the same order in the integral (8) defining the mean delay $\bar{z}$.

The first three model approximations listed above will limit the quantitative accuracy but not the qualitative results of the present study. Universality arguments indicate that the critical exponents, such as $\nu$ (which describes correlation lengths and finite-size effects) and $\beta$ (which describes $P(u)$ ) will not be affected by such local details of the model. The amplitudes involved in the various critical phenomena will depend on the structure of the lattice. However, most of this dependence will involve gross features such as the mean number of neighbours for which the present model seeks to match observational data as closely as possible. 

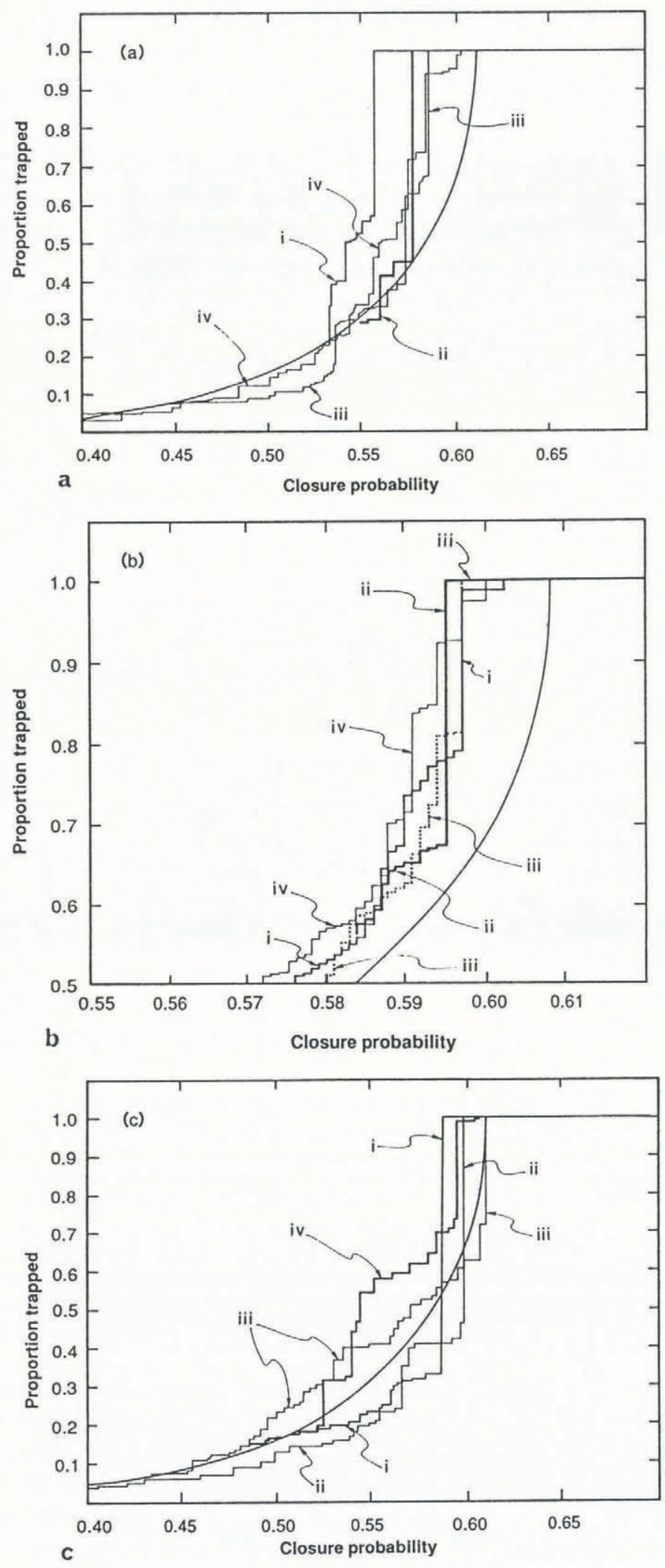

Fig. 2. Realizations of $S(u)$, the proportion of trapped bubbles, for finite samples of bubbles, plotted as functions of bond-closure probability, $u$. The smooth curve in each case is the infinite lattice estimate obtained from series expansions. Three or four realizations are shown in each case; the small roman numerals are a guide to distinguishing the lines. Some cases are only shown in part because of a high degree of overlap with other cases.

(a) Case 1: sample of 324 sites from lattice of 4752 sites;

(b) Case 3: sample of 20736 from 290304 sites; (c)

Case 5: sample of 324 sites from 36228 sites.
For the purposes of the simulation, the $u_{i}$ and $u_{i j}$ were discretized at intervals of 0.0001 and represented by 16 bit integers. The plots of $S(u)$ are based on values tabulated as intervals of 0.001 . In relating our simulations to actual ice cores, we have used estimates of 260 bubbles per cubic centimetre based on analysis of photographs of sections from the "ice" region of the BHD core analysed by Pearman and others (1986). We have analysed our results as though the closure probability $u$ was directly proportional to $z$. This is interpreting the intuitive description, given above, of the $u_{i}$ and $u_{i j}$ as times as literally true but it is a fairly crude approximation. The series-based estimate of the percolation probability $P$ on the lattice of b.c.c. interstitial sites was obtained by Enting $(1986,1987)$. The estimate $1-P$ is plotted as the solid curve in Figure 2 and suggests that the change from $10 \%$ closed off to $90 \%$ closed off occurs over a period of $\Delta u=0.142 \approx 0.23 u_{\mathrm{c}}$ while for the BHD core the change from $10 \%$ to $90 \%$ closure occurs over about $\Delta z \approx 14$ years $\approx 0.2 z_{\mathrm{c}}$.

In order to relate the simulations more closely to actual ice samples, the points of $10 \%$ and $90 \%$ closure give a better basis for matching the scales of age $z$ and closure probability, $u$. Thus, ranges expressed in terms of $u$ should be converted to time ranges by multiplying by $\Delta z / \Delta u$ with $\Delta z$ determined for the particular core.

We have performed a sequence of simulations for various sizes of lattice and the sample sub-set $A$. As noted in section 2, there are two different types of finite-size effect involved. The first of these, which has been most extensively studied by other workers, is the effect of using a finite size of lattice for the simulations. For the present study, this effect represents a source of error that must be taken into account when analysing the simulations. The second finite-size effect, in which we are primarily interested, is the degree of statistical variability that arises from using finite samples of bubbles. This is a question of sampling statistics, a topic that has not often been considered in lattice statistics. In order to estimate the importance of the two finite-size effects separately, we

Table 1. Details of sizes of lattices and samples used in the simulations. The lattices always had a square cross-section with periodic boundary conditions in the horizontal plane. The samples used to determine the statistics were always cubes. All dimensions are given in terms of the cubic cell containing 12 sites, except that the sample size is given as the number of sites

\begin{tabular}{rrrrrrr} 
Case & $\begin{array}{c}\text { Lattice } \\
\text { depth }\end{array}$ & $\begin{array}{c}\text { Lattice } \\
\text { width }\end{array}$ & $\begin{array}{c}\text { Sample } \\
\text { width }\end{array}$ & $\begin{array}{c}\text { Sample } \\
\text { top }\end{array}$ & $\begin{array}{c}\text { Sample } \\
\text { bottom }\end{array}$ & $\begin{array}{c}\text { Sample } \\
\text { size }\end{array}$ \\
\hline & & & & & & \\
1 & 13 & 6 & 3 & 7 & 9 & 324 \\
2 & 23 & 12 & 6 & 11 & 16 & 2592 \\
3 & 44 & 24 & 12 & 20 & 31 & 20736 \\
4 & 86 & 48 & 24 & 38 & 61 & 165888 \\
5 & 23 & 6 & 3 & 12 & 14 & 324 \\
6 & 44 & 24 & 3 & 25 & 27 & 324 \\
\hline
\end{tabular}


have varied the lattice size and the sample size independently, considering the various cases listed in Table 1.

The samples have their linear dimensions increasing by a factor of 2, and involve 324, 2592, 20736 and 165888 sites. For comparison, we estimate that the $1 \mathrm{~kg}$ samples analysed in our laboratory contained about 290000 bubbles (on the basis of 260 bubbles $\mathrm{cm}^{-3}$ and a density of $0.9 \mathrm{Mg} \mathrm{m}^{-3}$ ). Stauffer and others (1985) estimated that the $35 \mathrm{~cm}^{3}$ samples used in their work and that of Schwander and Stauffer (1984) contained about 30000 bonds (i.e. 15000 sites on the ultramarine lattice). Earlier studies by the Bern group used samples as small as $1 \mathrm{~g}$ which would correspond to about $400-500$ bubbles.

\section{RESULTS}

The three parts of Figure 2 each show four realizations of $S(z)$ for cases 1,3 and 5 of Table 1 . Also shown, as the smooth line on each figure, is the estimate of $S(z)$ derived from the series expansions given by Enting (1986). The estimate is expressed as $1-\left[V_{8}(u) / W_{7}(u)\right]^{0.454}$ where $V_{8}(u)$ and $W_{7}(u)$ are polynomals of degree 8 and 7 , respectively. Specifically, $V_{8}(u) / W_{7}(u)$ is the Pade approximant to $P(u)^{1 / 0.454}$ obtained from the series for $P(u)$ given by Enting (1986).

Figure $2 \mathrm{a}$ and $\mathrm{b}$ (cases 1 and 3 ) illustrate a systematic tendency (confirmed by the results of cases 2 and 4 ) for the simaller samples to produce small values for the cut-off probability so that, as the sample-size increases, the cutoff tends towards the series estimate. This size dependence is primarily due to the finite size of the lattice used. Comparing Figure $2 \mathrm{a}$ and $\mathrm{c}$ shows that the cut-off probabilities for the smallest samples tend to increase as the size of the surrounding lattice increases. The degree of variability between the realizations remains essentially unchanged. This size dependence of the average cut-off seems to explain much of the discrepancy between the values of $u_{c}$, estimated from series techniques as $0.61 \pm 0.01$ (Enting, 1986), and from Monte Carlo simulations of up to 8748 bonds, as 0.58 (Stauffer and others, 1985).

The various parts of Figure 2 show the variability (and size dependence of this variability) in the cumulative trapping distribution as a whole. As noted above, in studies of gas trapping, we are primarily concerned with the mean trapping time, $\bar{z}$, defined by Equation (6), and wish to determine how much sample-to-sample variation can occur in this quantity. It is to be expected that the mean trapping time will be less variable than the various aspects of $S(z)$ because it can be defined as an integral over $S(z)$.

However, it must be emphasized that for any single sample, the deviations between $S(z)$ and its ensemble mean are not independent for various $z$ values. In particular, the deviations must be such that $S(z) \rightarrow 1$ for sufficiently large $z$. Indeed, the need to construct quantities based on the closure history of single samples was an important reason for using Monte Carlo simulation in this study. Alternative approaches such as using statistics of cluster size to determine finite-size effects would produce ensemble averages at each $u$ value rather than statistics describing the closure histories of individual samples.

Figure 3 shows the cumulative distributions of the mean trapping times based on 20 realizations for the three smallest cases. It will be seen that, except for the smallest (324 site) examples, the distribution of trapping times is small compared to the mean time over which closure takes place. These results indicate that the mean closure probability is relatively insensitive to the critical fluctuations that affect finite-size samples.

Finally, we consider the scaling properties of the distribution of trapping times shown in Figure 3. For 20 $L \times L \times L$ samples, we order the 20 mean trapping times $\bar{z}_{j}$ and, as a robust measure of the spread, consider the

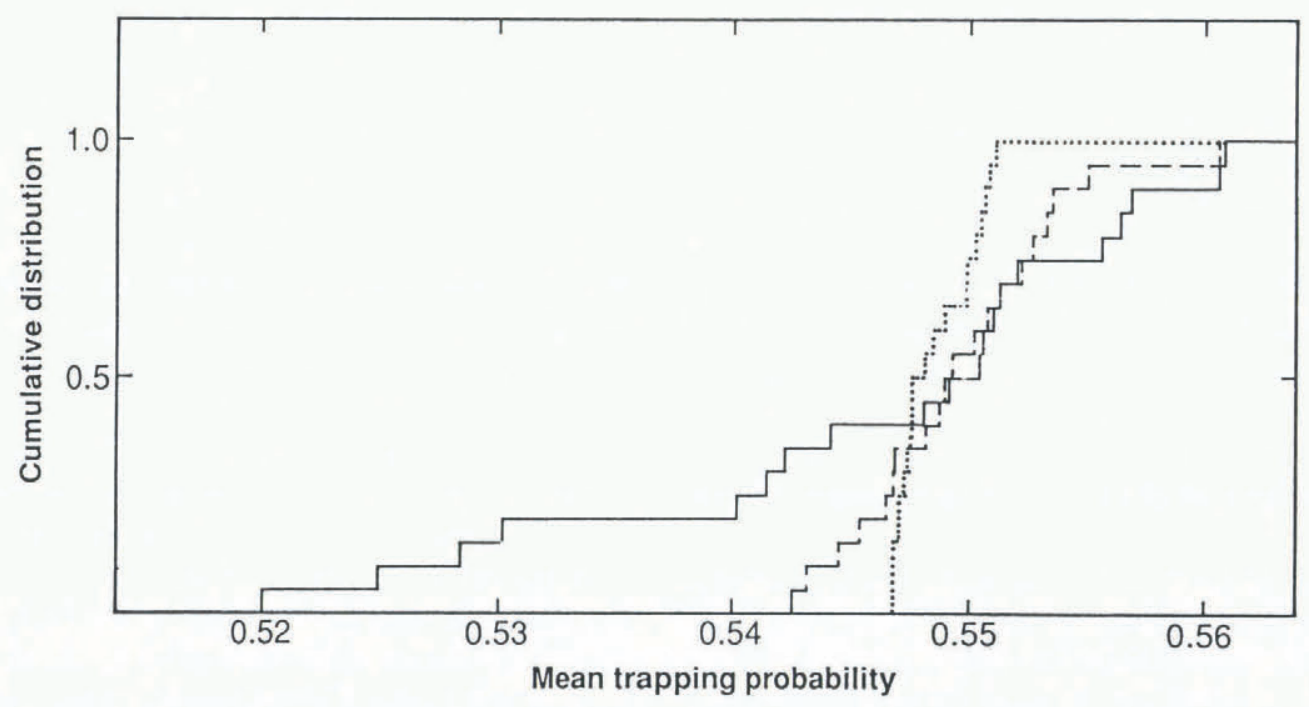

Fig. 3. Cumulative distributions of mean trapping times obtained from 20 realizations each of cases 1,2 and 3. Solid curve, case 1; dashed curve, case 2; dotted curve, case 3. 


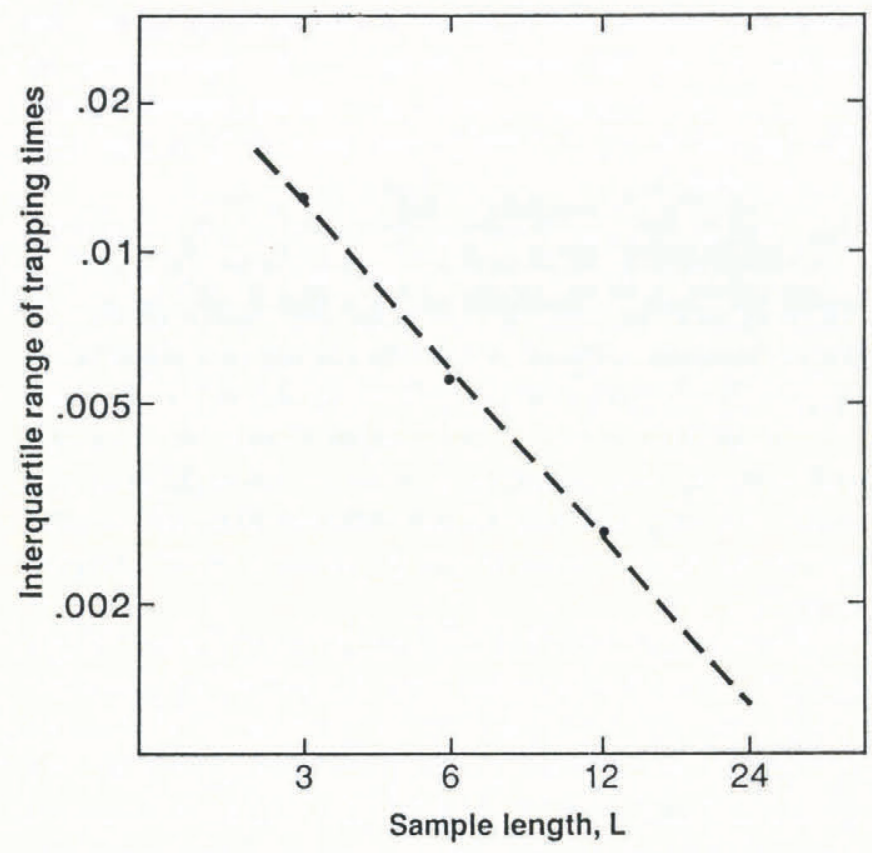

Fig. 4. Interquartile range of the mean trapping time plotted against sample length $L$. The results were from the sets of 20 cases used to produce Figure 3. The scaling line $a L^{-l / v}$ with $v=0.9$ is shown dashed.

interquartile range $\tau=\left(\bar{z}_{16}+\bar{z}_{15}-\bar{z}_{6}-\bar{z}_{5}\right) / 2$. These are shown on a logarithmic plot against $L$ in Figure 4. Finitesize scaling would suggest $\tau \sim L^{-1 / \nu}$ with $\nu \approx 0.9$ in three dimensions. The scaling line is shown on Figure 4 and is consistent with the data, although our samples are too small to give a precise estimate of $\nu$. What is clear is that $\tau$ is decreasing far more slowly than the $L^{-D / 2}$ (with $D=3$ ) that would occur for independently trapped bubbles. This emphasizes the co-operative nature of the bubble-trapping process.

\section{EXTENSIONS}

The analysis above has described the effect of finite sample size on the statistics of bubble-trapping time. Transferring these results to the statistics of gas age is only valid when the trapping time is long compared to the mixing time through the firn. For cores from areas of even moderately high snow accumulation this condition will cease to be valid.

Schwander (1988) has performed some modelling studies of the cases where the effective gas age is influenced by the slowness of gas mixing through the firn. The firn properties were determined by laboratory measurements. The percolation model can shed further light on this problem of diffusive mixing through the firn near close-off.

The relevant problem in percolation theory has been studied in terms of the problem of conductivity of random resistor networks. The conductivity is the analogue of the diffusivity in a network of channels. The conductivity problem in percolation theory is a matter of some difficulty. There is apparently no series-expansion formalism for calculating the conductivity, although related statistical moments can be calculated and the critical behaviour of the conductivity can be inferred from these. It is also possible to determine the conductivity from Monte Carlo experiments, either by the tedious "brute-force" method of solving Kirchoff's equations on a set of random networks, or more elegantly through a "self-averaging" transfer-matrix method described by Derrida and others (1984). The result is that, as expected, the conductivity goes to zero at the critical point. Most importantly for the ice-core application, the critical exponent is greater than 1 so that the gradient of the conductivity curve is zero at the critical point, in contrast to $S(z)$ where the gradient is infinite. This means that the conductivity in a resistor network (and diffusivity in a network of channels) may be small throughout a significant fraction of the critical region and possibly beyond.

These properties of the conductivity/diffusivity problem have important implications for gas dating, depending on the relative time-scales of diffusion and bubble closure. If the bubble trapping is slow relative to diffusive mixing, then only the trapping distribution need be considered. For somewhat faster trapping, the effect of restrictions on the diffusive mixing will be to produce an age distribution that is more rounded than the trapping distribution. This will reduce the extent to which changes in atmospheric composition can be resolved from the icecore record.

The most significant effects of restrictions on diffusion occur when differences between summer and winter accumulation lead to "winter" layers sealing off the "summer" layers below them even though the "summer" layers may be still permeable and have only a small fraction of bubbles trapped by local closure. In such a case, it would be expected that the statistical variability of trapping in "summer" layers is largely due to variations in the overlying winter layer and so much of the variability will involve year-to-year differences rather than within-year differences. The sample size becomes a relatively unimportant issue. In contrast, within the "winter" layers where the effective closure is due to changes within that layer, local statistical fluctuations are likely to play a much more important role.

\section{GONGLUSIONS}

The simulations described in this paper were carried out in order to assess the influence of critical fluctuations in the firn-closure process on the dating of gas samples trapped in polar ice. Since it has been possible to perform simulations of systems whose size is comparable to those of actual interest, it has not been necessary to use finite-size scaling to extrapolate the results of the simulation.

The main result of these studies is that the mean trapping time $\bar{z}$ is relatively independent of sample size over the range of interest. Furthermore, over this range, the sample-to-sample variation in the $\bar{z}$ is largely confined to a range that is small compared to the time over which most of the closure (firnification) takes place, except for small samples of order $1 \mathrm{~g}$. However, the simulations also suggest that for gases with rapid changes in atmospheric concentrations (and for which the mean trapping time is 
not applicable) critical fluctuations may significantly affect the trapped gas concentrations.

The simulations suggest that the one important way in which critical fluctuations will affect gas dating is through the process of relating closure properties to actual ages. The conventional specification of this relation is to determine times at which $10 \%$ and $90 \%$ of the trapping has occurred. The simulation results shown in Figure 2 indicate that there can be a great deal of sample-tosample variation in these two times, arising purely from the inherent statistical variability of the trapping process. The variation in the $90 \%$ trapping point is larger than the interquartile range for the respective sample sizes. Near the $u$ values corresponding to $10 \%$ trapping, there is much less variability in the trapped volume but the flatness of the trapping distribution makes the position of the $10 \%$ point rather uncertain. This "calibration" problem will be exacerbated by the fact, noted above, that the critical region is one in which the system will be highly susceptible to the influence of external perturbations. As discussed above in connection with the effect of external perturbations, an uncertainty in the timing of the trapping due to the "calibration" will lead to pointwise values of $S(z)$ being subject to large uncertainties. It turns out that $\bar{z}$ does not reflect this sensitivity; the uncertainty remains comparable to the uncertainty in the timing of the trapping.

\section{ACKNOWLEDGEMENTS}

The author wishes to thank G. R. Enting for the use of computing equipment for the development of the programs used in this study, and the Mathematics Department of Melbourne University for the use of computing facilities for the larger cases. Helpful comments by J. V. Mansbridge, A. C. Hirst, H. Granek, D. Etheridge and D. Raynaud are gratefully acknowledged. In attempting to bridge the gap between the fields of lattice statistics and glaciology, I have been greatly assisted by the comments of the referees.

\section{REFERENCES}

Barnola, J. M., D. Raynaud, Ye.S. Korotkevich and C. Lorius. 1987. Vostok ice core provides 160,000-year record of atmospheric $\mathrm{CO}_{2}$. Nature, 329(6138), 408414.

Delmas, R.J., J.-M. Ascencio and M. Legrand. 1980. Polar ice evidence that atmospheric $\mathrm{CO}_{2} 20,000 \mathrm{yr}$ BP was $50 \%$ of present. Nature, 284(5752), 155-157.

Derrida, B., J. G. Zabolitzky, J. Vannimenus and D. Stauffer. 1984. A transfer matrix program to calculate the conductivity of random resistor networks. $\mathcal{F}$. Stat. Phys., 36, 31-42.

Efros, A. L. 1986. Physics and geometry of disorder: percolation theory. (Translation from Russian by V.I. Kisin. Moscow, Mir Publishers.)

Enting, I. G. 1985a. A classification of some inverse problems in geochemical modelling. Tellus, 37B, 216229.

Enting, I. G. 1985b. A lattice statistics model for the age distribution of air bubbles in polar ice. Nature, 315(6021), 654-655.

Enting, I. G. 1986. Lattice models of firn closure: I. Percolation on the interstices of the BCC lattice. 7 . Phys. A, 19(14), 2841-2854.

Enting, I. G. 1987. On the application of lattice statistics to bubble trapping in ice. Tellus, 39B(1-2), 100-113.

Enting, I. G. and J. V. Mansbridge. 1985. The effective age of bubbles in polar ice. PAGEOPH, 123, 777-790.

Fortuin, C. M. and P.W. Kasteleyn. 1972. On the random cluster model. I: Introduction and relation to other models. Physica, 57, 536-564.

Loosli, H.H. 1983. A dating method with ${ }^{39}$ Ar. Earth Planet. Sci. Lett., 63, 51-62.

Mandelbrot, B. B. 1977. Fractals: form chance and dimension. San Francisco, CA, W.H. Freeman.

Neftel, A., E. Moor, H. Oeschger and B. Stauffer. 1985. Evidence from polar ice cores for the increase in atmospheric $\mathrm{CO}_{2}$ in the past two centuries. Nature, 315(6014), 45-47.

Pearman, G. I., D. Ethridge, F. de Silva and P.J. Fraser. 1986. Evidence of changing concentrations of atmospheric $\mathrm{CO}_{2}, \mathrm{~N}_{2} \mathrm{O}$ and $\mathrm{CH}_{4}$ from air bubbles in Antarctic ice. Nature, 320(6059), 248-250.

Schwander, J. 1988. The transformation of snow to ice and the occlusion of gases. In Oeschger, H. and C. C. Langway, $\mathrm{Jr}$, eds. The environmental record in glaciers and ice sheets. Chichester, etc., Wiley, 55-67.

Schwander, J. and B. Stauffer. 1984. Age difference between polar ice and the air trapped in its bubbles. Nature, 311(5981), 45-47.

Schwander, J., B. Stauffer and A. Sigg. 1988. Air mixing in firn and the age of the air at pore close-off. Ann. Glaciol., 10, 141-145.

Stauffer, B. 1982. Mechanismen des Lufteinschlusses in natürlichem Eis. Z. Gletscherkd. Glazialgeol., 17(1), 1981, 17-56.

Stauffer, B., J. Schwander and H. Oeschger. 1985. Enclosure of air during metamorphosis of dry firn to ice. Ann. Glaciol., 6, 108-112.

Stauffer, D. 1985. Introduction to percolation theory. London, Taylor and Francis.

Wu, F.Y. 1978. Percolation and the Potts model. 7 . Statist. Phys., 18, 115-123.

Zallen, R. 1983. Percolation: a model for all seasons. In Deutscher, G., R. Zallen and J. Adler, eds. Percolation structures and processes. Bristol, Adam Hilger, 3-16.

The accuracy of references in the text and in this list is the responsibility of the author, to whom queries should be addressed.

\section{APPENDIX}

\section{DETAILS OF THE SIMULATION}

In view of the potential for applying the model to simulations of other statistical aspects of firn closure, this Appendix gives additional details of the simulation procedure.

As noted in the body of the paper, we model the firn using the b.c.c. (body-centred cubic) lattice to represent the snow grains with the interstitial sites representing the 
locations of air bubbles. We describe the lattice in terms of cubic cells containing 12 sites and 24 bonds as shown in Figure 1. In order to avoid duplication in the scanning process, the bonds are associated with the sites of even indices, as indicated by the arrows in Figure 1. We consider arrays of $m \times m \times n$ of these cubic cells. We apply periodic boundary conditions in the horizontal $(m \times m)$ layers and consider the statistics of connection to the upper boundary (layer 1 ). The simulation procedure assigned to each bond (connecting sites $i$ and $j$ ) a cut-off level $u_{i j}$ which corresponded to the value of the probability level $u$ at which the bond $(i, j)$ was regarded as closed.

The model statistics of bubble trapping were obtained from the site-closure values $u_{i}$ that represented the value of $u$ above which the site $i$ was no longer connected to the surface. Thus

$$
u_{i}=\max _{\text {all paths } \alpha_{i}}\left[\min _{\text {bonds }(j, k) \in \alpha_{i}}\left(u_{j k}\right)\right]
$$

where each path $\alpha_{i}$ is a chain of connected bonds from site $i$ to the upper boundary. The topmost (layer 1) and bottommost (layer $n$ ) layers were dummy layers which were not analysed specifically but which provided boundaries so that each of the $n-2$ active layers was surrounded by two layers. The $u_{i}$ were initialized to 1 in layer 1 and to zero in all other layers. The $u_{i j}$ were set to 1 in layer 1, 0 in layer $n$ and pseudo-random numbers (selected uniformly from the range $[0,1]$ in the $n-2$ active layers to represent the random times of bond closure. The $u_{i}$ were calculated iteratively by repeatedly applying the transformations

$$
\begin{aligned}
& u_{i} \leftarrow \max \left(u_{i}, \min \left(u_{j}, u_{i j}\right)\right) \\
& u_{j} \leftarrow \max \left(u_{j}, \min \left(u_{i}, u_{i j}\right)\right)
\end{aligned}
$$

for all the bonds $(i, j)$ until no further changes occurred. The scanning procedure worked through the sites of even index (see Fig. 1) in each cell in the $n-2$ active layers and considered each of the four bonds in turn, updating sites at either end of the bond as appropriate. This procedure will update a small number of sites (of types 1 and 3 ) in layer $n$ and will neglect a small number of sites in layer 2. (In other words, the effective upper and lower boundaries are not exactly planar.)

In practice, as the iteration evolved, almost half of the bonds could be removed from further consideration because they could not be part of any current or future maximal path. The criterion for this removal was

$$
u_{i j}<u_{i} \quad \text { and } \quad u_{i j}<u_{j} .
$$

The further removal of bonds for which

$$
u_{i j} \leq u_{i} \quad \text { and } \quad u_{i j} \leq u_{j}
$$

was posssible because, although bonds satisfying (A.3b) but not (A.3a) were part of some maximal path, once (A.3b) was satisfied the states of the sites reflected this fact. Applying the transformations (A.2a and b) will not change either $u_{i}$ or $u_{j}$ once (A.3b) holds, even if either $u_{i}$ or $u_{j}$ subsequently changes. The bubble-trapping statistics were computed using a sub-set, $A$, of sites.

As noted in the body of the paper, the bubbletrapping distribution was

$$
S(u)=|A|^{-1}|X(u)|
$$

where

$$
X(u)=\left\{u_{i}: i \in A \quad \text { and } \quad u_{i} \leq u\right\} .
$$

This model can form the basis for refined calculations such as those which include the role of gas in the channels and the effects of density on the mean bubble size. However, it cannot be readily adapted for the more significant question of analysing the restriction on transport through the firn as the transition is approached. For this problem, the algorithm of Derrida and others (1984) would seem to be more suitable. 